\title{
Linguostylistic Peculiarities of US Medical Leaflets
}

\author{
Naira Gasparyan \\ Yerevan State University
}

$\mathrm{M}^{\mathrm{c}}$ edical leaflets present a genre belonging to the domain of scientific documents where two major functional varieties are interwoven to present a specific piece of text wrought in a strict documentary form and saturated with scientific terms.

Although medical leaflets are meant for masses and not specifically for medical people, the amount of terms is still high here, but as compared to the literature specifically oriented towards medical professionals, the number of terms in leaflets is relatively low. Research has established that the vocabulary in scientific medical texts is dispensed in a different way as compared to the average scientific piece of text. Actually in medical texts the words belonging to General English have the least amount, while special medical terms cover the greatest part of the vocabulary. In the majority of cases the intended reader of medical leaflets is the casual citizen, and the content of the leaflet (how to use the drug in every particular case) is explained to the user by a medical worker: either by the doctor who writes the prescription, or by a medical advisor in the drug store.

Any medical leaflet, irrespective of the country it is compiled in, strictly meets certain linguistic requirements based on the extra-linguistic reality of that very country. The US medical leaflet is compiled according to a scheme almost similar to a leaflet of any other country, but visually it has a bigger volume because it includes extra columns. These columns presenting such details as information about the drug tests on animals and humans, the corresponding Patent information as well as FAQs or patient information are left out in the leaflets of other countries. Since the message of the last section in a US leaflet is oriented towards the average and even less than the average member of the society, it is transmitted to the addressee through:

1. the vocabulary of general English, with the minimum use of scientific medical terms and;

2. complicated grammatical patterns, like parallel constructions including stylistic repetition and/or enumeration, that can be understood without much effort, hence, making the content of the message available and open to perceive.

The most complicated piece of the text from the point of both grammar and vocabulary is the patent information since the addressee here is the medical specialist and not the average citizen. Being a medical official document, leaflet texts are deprived of emotional-evaluative overtones. The addressee orients a message which carries certain instruction appeals to the reader immediately and involves him directly into the process of treatment.

The form-function relationship manifesting itself on the firm ground of instructing makes the document a specific genre where the directive language function is realized through a variety of expression aiming at either causing or preventing action. Negative behavior is warned and dangerous situations are mentioned. Although Imperative mood is used in such cases, the verb modal should affirmative or negative (should not) can 
appear to indicate warning. The directive function seems to be domineering in this type of a text at first sight, but there is the opinion that when one function is accentuated, it tends to diminish the importance of all the others. Yet, there is another opinion that some functions are generally paired in an even more definite relation.

Thus, in the material of our investigation, as the observations show, the conative function, which aims at appealing to the addressee's mind and will in order to exhort and persuade him, is the actual pair of the directive function and is realized with the help of the modal should, Present Simple negative and imperatives.

Our observation has also revealed that paragraphs of the whole document of the leaflet are arranged in a fixed structure establishing a thematically and logically coherent sequence where not only the directive-conative pair, but also the referential-informative, phatic-communicative, and the cognitive functions are realized to secure the official and medical value of the document. The referential-informative pair of functions, oriented toward the context, is realized through certain lexical (terms) and grammatical (Present Simple, Past Simple, The Present Perfect, the Passive Voice, the modal may, etc.) phenomena.

The phatic-communicative pair of functions is realized through establishing a certain communication between the addressor and the addressee. What matters here is the construction of the text of the leaflet. Naturally, before coming to the part where the dosage and instructions are discussed, the leaflet defines the medicine presenting some vitally important, precise and trustworthy information about its physical and chemical properties, then passes on to the health problems it can cure and, finally, the possible symptoms of the instances are described. Obviously, these parts of the text prepare the patient and/or the caretaker to perceive the importance of the use of that very drug, and if the patient is successfully persuaded that the drug can help to effectively handle that particular case, he will immediately follow the instructions. Certain passages especially of patient and patent information and of the core text as well, including paragraph heads, are realized through certain cognitive constructions which will be discussed within the frames of the present work.

The directive function realized on a firm basis, is the head while the other above-mentioned functions serve as a body, and they all act together as parts of a whole.

Now we intend to establish the extra-linguistic factors that have their fixed impact on leaflet texts.

The US government has set targets for the supply of useful written information to patients when they get their first supply of medicine. The law defines this useful written information as being scientifically accurate, non-promotional, specific and comprehensive, understandable and legible. Then meeting the society's demand, in 1996, it was decided that People who suffer should help write leaflets ${ }^{1}$. This is how the patient information section appeared. It was also settled that leaflets should have a plain language, good design, and describe the degree of any risk. Actually this is what can be called the extra-linguistic reality for the US medical leaflets.

Now we'd like to single out those lexical and grammatical peculiarities that successfully convey the addressor's message and can be viewed as cognitive constructions (the leaflets are written and produced by third parties, such as US Pharmacopeia, American Medical Association, American Society of Health System). 
The functions of language realized on the mentioned extra-linguistic basis determine the linguostylistic peculiarities of the texts of medical leaflets. We have singled out the following groups of the mentioned peculiarities:

1. The following syntagms noun+noun; noun+and+(adj.)+noun and adj.+noun appear to be cognitive constructions for this specific type of a text as they introduce thematic paragraphs, e.g.

noun+noun: System Organ Class, Adverse Reaction, Adverse Disorders, Gastrointestinal Disorders.

Noun/adj.+and+(adj.)+noun: Renal and Urinary Disorders, General Disorders and Administration Site Conditions, Reproductive and Breast Disorders, Male and Female Disfunctions, Indication and Usage.

2. Single nouns also can appear to be used as carriers of a certain message, like Warnings, Precautions, Overdosage, Description.

3. ...of... constructins are another peculiarity of the document as in Management of Overdose, Mechanism of Action.

4. Latin phrases like in vivo (is experimentation using a whole, living as opposed to a or dead organism); in vitro (within the glass, i.e., in a test tube or a petri dish).

5. The Use Of Acronyms, which have their corresponding full variants used for the first time in the text of that particular leaflet, like NSAID (Non steroidal and inflammatory drug), BP (Blood Pressure), $C V$ (Cardiovascular), SJS (Stevens-Johnson Syndrome), TEN (Toxic epidermal necrolysis), GI (Gastrointestina), CABG (Coronary artery bypass graft).

6. The use of Modals should, can, may: An airway should be established; Indometacin should not be given to patients who have experienced asthma; Patients taking thiazides or loopdiuretics may have impaired response to these therapies...; Blood pressure (BP) should be monitored closely...; Indometacin may cause serious side effects...; Acute overdosage with tramadol can be manifested by respiratory depression.

7. The use of Present (active and passive) and Past Simple, Present Perfect: ULTRAM ER is not recommended for obstetrical preoperative medication; Gender did not influence Atomoxetine disposition; Atomoxetine did not effect...; Lisinopril has been found to be generally well tolerated in controlled clinical trials....

8. Negative constructions introduced by NO: No fetal effects were seen when pregnant rats were treated with..; No adequate and well controlled studies have been conducted in pregnant women.

9. Inversion introduced by the preposition IN: In vitro drug-displacement studies were conducted with Atomoxetine; In 1 or 2 studies, decreases in pup weight and pup survival were observed (rats); In late pregnancy as with other NSAIDs, Indometacin should be avoided.

10. Anaphorial Parallel constructions forming periods with stylistic repetition: NSAID medicines may increase the chance of heart attack or stroke that can lead to death. The chance increases: With longer use of NSAID medicines in people who have heart disease. 
NSAID medicines should never be used right before or after a heart surgery called a CABG.

NSAID medicines can cause ulcers and bleeding in the stomach and intestines at any time during treatment.

NSAID medicines should only be used.

Summing up it can be said that American drug instructions are very specific as compared to those of European and Armenian ones and contain parts which are very often detached. Having an official documentary value, American drug instructions nevertheless do not contain officialese and are very essential for cognition. As far as the translation of leaflets is concerned, it should be mentioned that leaflets are professional documents and while translating them into Armenian certain rules are observed: the patient and patent information is omitted since Armenian Medical Law does not have any requirement concerning the availability of patient and patent information as well as FAQs. Actually the translation of the leaflet turns out to be a new text compiled according to the Law in action in Armenia.

\section{Note:}

1. The US Government has set targets for the supply of "useful written information" to patients when they get their first supply of medicine.

$<$ www.mcppnet.org/publications/ISSUE07-2.pdf $>$

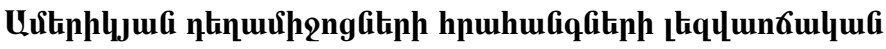 unufiåfiuhuunlnıpjnıfifitinn}

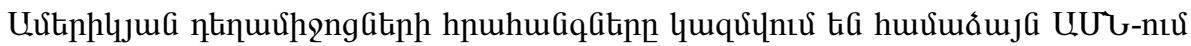

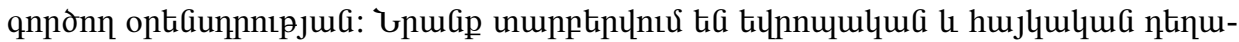

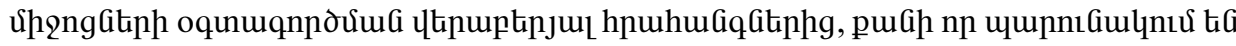

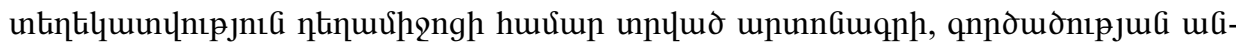

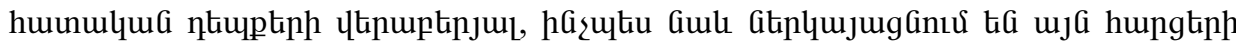

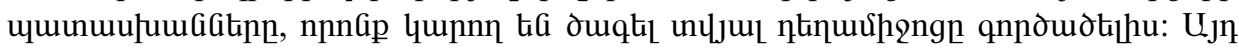

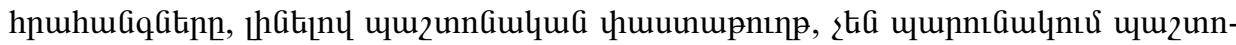

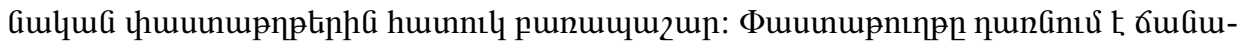

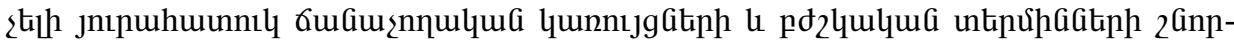

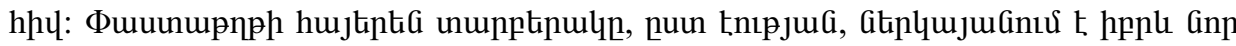

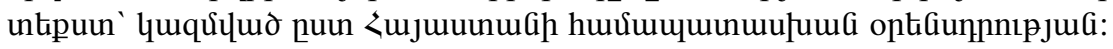

